International Mathematical Forum, Vol. 8, 2013, no. 24, 1185 - 1193

HIKARI Ltd, www.m-hikari.com

http://dx.doi.org/10.12988/imf.2013.3354

\title{
On the Geometry of $m$-Convex Sets in the Euclidean space
}

\author{
M. Beltagy \\ Mathematics Department, Faculty of Science \\ Tanta University, Tanta, Egypt \\ beltagy50@yahoo.com
}

S. Shenawy

Modern Academy for Engineering and Technology in Maadi

Maadi, Egypt

s.shenawy@s-math.org, sshenawy08@yahoo.com

drssshenawy@eng.modern-academy.edu.eg

\section{S. Mosa}

Faculty of Science, Damanhour University, Damanhour, Egypt. saffamosa@yahoo.com

Copyright (c) 2013 M. Beltagy et al. This is an open access article distributed under the Creative Commons Attribution License, which permits unrestricted use, distribution, and reproduction in any medium, provided the original work is properly cited.

\begin{abstract}
A set $A$ in Euclidean $n$-space $E^{n}$, is called an $m$-convex set if for every $m$ distinct points of $A$ at least one of the line segments joining two points of them lies in $A$. In this article we study some geometrical and topological properties of these sets in $E^{n}$.
\end{abstract}

Mathematics Subject Classification: 53C42, 52A05

Keywords: $m$ - convex sets, $p_{3}$ property, starshaped sets, extreme points, maximal convex sets, radial contraction 


\section{Introduction}

The notion of convexity is essential in both geometry and analysis. So, it has been generalized in many aspects and different reasons. In this article, we introduce some generalizations of this concept in $E^{n}$.

F.A. Valentine in [8], proved that for a closed connected 3- convex set $A$ in $E^{n}, A$ is either convex or is starshaped with respect to each of its points of local non-convexity. But this is not true for a closed connected 4- convex set. So, it is not generally true for an $m$ - convex set. Also, he proved that $A$ can be considered as the union of three or less closed convex sets having a non-empty intersection.

Also, M. Breen in [3], presented a similar decomposition without requiring the set $A$ to be closed. It is proved in this article that, a 3- convex set $A$ in $E^{n}$ is expressible as the union of at most two maximal subsets having a non-empty intersection ( kernel of $A$ ).

The notion of radial contraction is considered. M. Beltagy and S. Shenawy in [2], proved that for a non-empty subset $A$ of $E^{n}, p \in E^{n}$, and $\lambda \in(0,1)$, then $A$ is convex if and only if $C_{p}^{\lambda}(A)$ is. It is proved in this article that, $A$ is an $m$ - convex set if and only if $C_{p}^{\lambda}(A)$ is. We introduce also the concept of $m$ affinity, and proved that $C_{p}(A)$ is an $m$ - affine set if $A$ is an $m$ - affine set.

Also, we take in consideration some geometrical and topological properties for $m$ - convex sets such as the union of two $m$ - convex sets, the intersection of a 2-convex set and an $m$ - convex set, and the extreme points of an $m$ - convex set. For more properties of $m$ - convex sets, see $[4,5]$.

\section{Notations and Definitions}

Let $x, y$ be in $A$. The closed segment joining $x$ and $y$ is denoted by $[x y]$ where $(x y)=[x y] \backslash\{x, y\}$ denotes the open segment joining $x$ and $y$. The straight line determined by $x$ and $y$ is denoted by $L(x \rightarrow y)$.

We say that $x$ sees $y$ via $A$ if and only if $[x y]$ is contained in $A[2,3,5]$. $A$ is called starshaped if there exists some point $p$ in $A$ such that $p$ sees each point of $A$ via $A$. In this case we say that $A$ is starshaped relative to $p$ [5]. The set of all such points $p$ is called the kernel of $A$, and is denoted by $\operatorname{ker}(A)$ $[1]$. 
A set $A \subset E^{n}$ is called convex if for any two points $p$ and $q$ belonging to $A$, the entire segment joining $p$ and $q$ lies in $A[6,7]$. The set $\operatorname{ker}(A)$ is convex [2]. The convex hull of a set $A$ is the smallest convex set that contains $A$, and is denoted by $C H(A)$. Obviously, $C H(A)=A$, when $A$ is convex [1].

Let $A$ be a subset of the Euclidean $n$ - space $E^{n}$. The set $A$ is said to be $m$ - convex, $m \geq 2$, if and only if for every $m$ distinct points of $A$ at least one of the line segments determined by these points lies in $A[4,5]$. A maximal convex set of a set $A$ is a convex subset of $A$ which is not contained in another convex subset of $A$.

An extreme point of a set $A$ is a point of $A$ which is not interior to any segment with ends belonging to $A$. The set of all extreme points of $A$ is denoted by $E(A)$. For any convex set, the set remains convex if its extreme points are removed $[6,7]$.

A hyperplane $H$ in $E^{n}$ is a supporting hyperplane to $A$ if $H$ intersects the closure of $A$ and $A$ is contained in a closed side of $H$ [7]. A supporting hyperplane $H$ is called a locally supporting hyperplane at $p \in A$ if there exists a neighborhood $N$ of $p$ such that $N \cap A$ is supported by $H$ [7].

Let $A$ be a non-empty subset of $E^{n}$. For a fixed point $p \in A$ and a fixed real number $\lambda \in(0,1)$, we define the $\lambda$ - radial contraction of $A$ based at $p$, is denoted by $C_{p}^{\lambda}(A)$, as

$$
C_{p}^{\lambda}(x)=\lambda x+(1-\lambda) p, \quad x \in A
$$

The set $C_{p}^{\lambda}(A)$ is called the $\lambda$ - radial contraction of $A$ based at $p$. If $\lambda=\frac{1}{2}$, we get the midpoint contraction of $A$ about $p \in A$, denoted by $C_{p}(A)$, and it is the set of all points $\frac{1}{2}(p+x), x \in A$, i.e.,

$$
C_{p}(A)=\left\{y: y=\frac{1}{2}(p+x), \quad x \in A\right\}
$$

A set $A$ is said to be affine if $x, y \in A$ implies $\lambda x+(1-\lambda) y \in A$ for all real numbers $\lambda$, i.e., for each pair of different points of $A$, the straight line $L(x \rightarrow y)$ is in $A[2]$. A set $A$ is called an $m$ - affine set, $m \geq 2$, if and only if for every $m$ different points $x_{1}, x_{2}, \ldots, x_{m}$ in $A$, at least one of the straight lines $L\left(x_{i} \rightarrow x_{j}\right)$ is in $A, 1 \leq i, j \leq m$. It is clear that every affine set is an $m$ affine set. 


\section{The Results}

In this section we present the main results of this paper, and we begin with the following propositions.

Proposition 3.1 Let $A$ be an $m$ - convex subset in $E^{n}$. Then $A$ is an $(m+k)$ - convex set for every positive integer $k$.

Examples can be found to show that the converse is not generally true.

Proposition 3.2 The union of an $m$ - convex set and a convex set is at most $(m+1)$ - convex set. Also, their intersection is an $m$ - convex set.

Proof. Let $A$ be an $m$ - convex set, and $B$ be a 2 - convex set. First, consider $(m+1)$ points in $A \cup B$. Now, if two points are in $B$ say $x_{1}, x_{2}$, this implies that $\left[x_{1} x_{2}\right]$ is contained in $B$ and hence is contained in $A \cup B$. Otherwise, there exists $m$ points in $A$, this implies that $\left[x_{i} x_{j}\right], 1 \leq i, j \leq m$, is contained in $A$, since $A$ is an $m$ - convex set. Thus, $\left[x_{i} x_{j}\right]$ is contained in $A \cup B$. So, $A \cup B$ is an $(m+1)$ - convex set.

Second, suppose that $A \cap B$ is not an $m$ - convex set. Then there are $m$ points $x_{i}, 1 \leq i \leq m$ in $A \cap B$ such that $\left[x_{i} x_{j}\right]$ is not contained in $A \cap B$ for every $i$ and $j$. But $B$ is convex i.e., $\left[x_{i} x_{j}\right] \subset B$ for every $i$ and $j$. Hence, $\left[x_{i} x_{j}\right]$ is not contained in $A, 1 \leq i \leq m$, i.e., we get $m$ points $x_{i}, 1 \leq i \leq m$, in $A$ such that $\left[x_{i} x_{j}\right]$ is not contained in $A$ for $1 \leq i \leq m$. This is a contradiction since $A$ is an $m$ - convex set, and the proof is complete.

For example, at $m=3$, we get the intersection of a 3 - convex set and a 2convex set is a 3 - convex set, as shown in Figure 1.
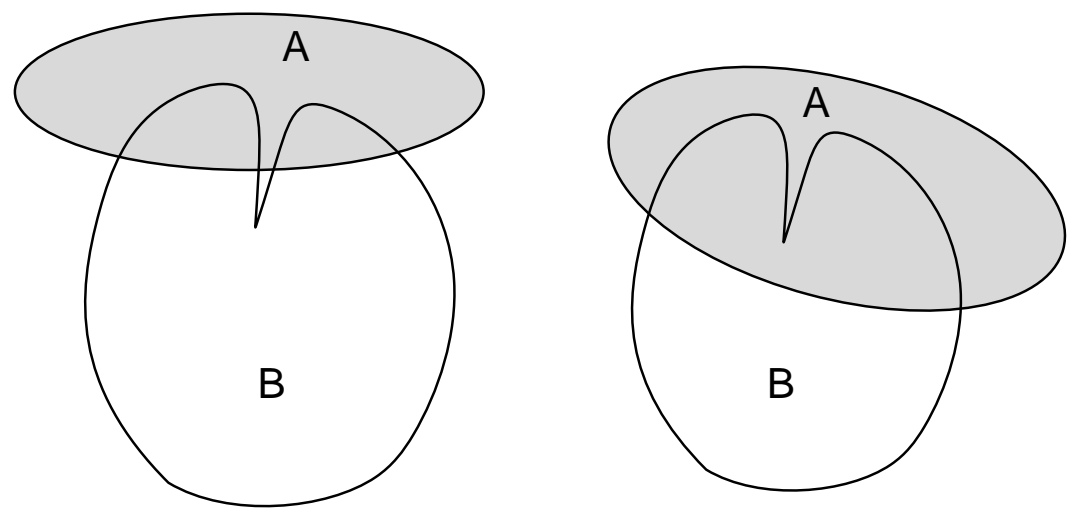

Figure 1: Intersection of a 3-convex set and a 2-convex set 
Remark 3.3 We can conclude that, the intersection of two $m$-convex sets is not generally $m$ - convex set. For example, the intersection of two 3convex sets is not a 3- convex set, as shown in Figure 2.

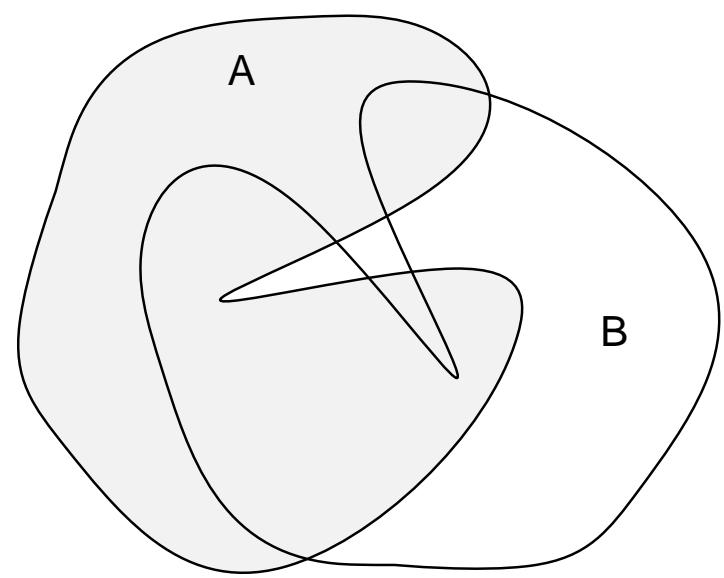

Figure 2: The intersection of two 3-convex sets is not generally 3-convex

Now, let $A$ be an $m$ - convex set and $B$ be a starshaped set. Then $A \cap B$ is not generally an $m$ - convex set. Let $A$ and $B$ be two $m$ - convex sets. Then it is proved that, in the following theorem, $A \cup B$ is not an $(m+1)$ - convex set but $A \cup B$ is an $(m+n)$ - convex set.

Theorem 3.4 Let $A$ be an $m$-convex set and $B$ be an $n$-convex set. Then $A \cup B$ is at most an $(m+n)$ - convex set.

Proof. Let $x_{1}, x_{2}, \ldots, x_{m+n}$ be $(m+n)$ points of $A \cup B$. If $m$ points or more of them are in $A$, then there is one segment $\left[x_{i} x_{j}\right] \subset A, 1 \leq i, j \leq m+n$ and hence $\left[x_{i} x_{j}\right] \subset A \cup B$. This implies that $A \cup B$ is an $(m+n)$ - convex set. If $A$ does not contain $m$ points of the points $x_{i}$ 's, then $B$ contains at least $n$ points of them and by the same way we get that $A \cup B$ is an $(m+n)$ - convex set, and the proof is complete.

Theorem 3.5 Let $A$ be a set having at most $(m-1)$ maximal convex subsets. Then $A$ is an $m$ - convex set.

Proof. Let $A$ be a set having at most $(m-1)$ maximal convex subsets $A_{r}$, $1 \leq r \leq m-1$, i.e., $A=\bigcup_{r=1}^{m-1} A$. We want to prove that $A$ is an $m$ - convex set. So, let $x_{1}, x_{2}, \ldots, x_{m}$ be $m$ distinct points of $A$. At least two points $x_{i}, x_{j}$, $1 \leq i, j \leq m$ lie in the same set $A_{r}$ and hence $\left[x_{i} x_{j}\right] \subset A_{r} \subset A$, i.e., $A$ is an $m$ - convex set. 
Remark 3.6 1. It is clear that, if $A$ is connected, then the intersection of all maximal convex subsets of $A$ is not empty, and hence $A$ is starshaped and $\operatorname{ker}(A)=\bigcap_{i=1}^{m-1} A_{i}$.

2.If $A$ is not connected, we get at most $(m-1)$ pieces and if the pieces are exactly $(m-1)$, then every piece is convex. The word convex is essential, as shown in Figure 3.

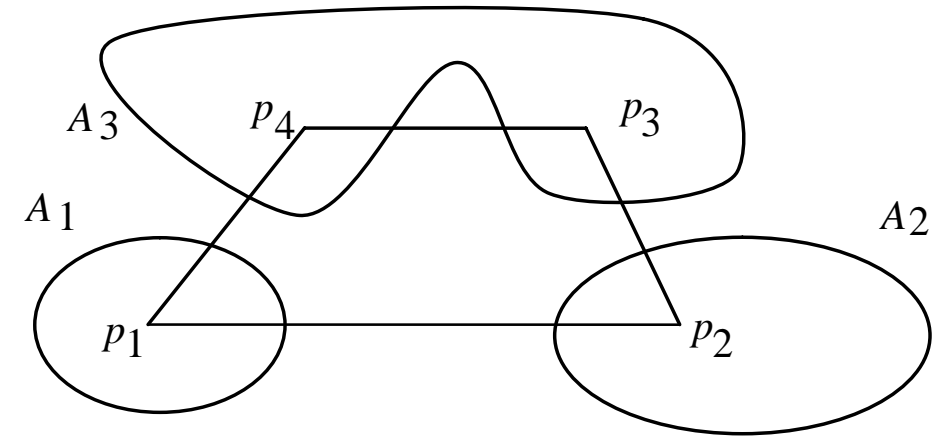

Figure 3: Convexity of each piece for a 4-convex set

Corollary 3.7 The union of $m$ disjoint convex sets $A_{i} \subset E^{n}, 1 \leq i \leq m$ is an $(m+1)$ - convex set.

Theorem 3.8 Let $A \subset E^{n}$ be an $m$ - convex set. If $p \in A$ is an extreme point, then $A \backslash\{p\}$ is an $m$ - convex set.

Proof. Suppose that $p \in E(A)$, where $E(A)$ is the set of all extreme points of $A$, and let $x_{1}, x_{2}, \ldots, x_{m}$ denote any $m$ distinct points of $B=A \backslash\{p\}$. Thus $x_{i} \neq p$ for all $1 \leq i \leq m$, and $x_{i}$ are points of $A$. Since $A$ is an $m$ convex set, then at least one of the segments $\left[x_{i} x_{j}\right] \subset A, 1 \leq i, j \leq m$. Since $p \in E(A)$, i.e., $p$ does not lie between any two points of $A$, and hence $p$ does not belong to $\left(x_{i} x_{j}\right)$. Hence $p$ does not belong to $\left[x_{i} x_{j}\right]$. Therefore, the segment $\left[x_{i} x_{j}\right] \subset B=A \backslash\{p\}$. So, $B=A \backslash\{p\}$ is an $m$ - convex set.

Remark 3.9 Examples can be found to show that the converse of the previous theorem is not generally true.

F. A. Valentine [8] proved that a closed connected 3- convex set $A$ in $E^{n}$ is either convex ( has no points of local non-convexity ) or is starshaped with respect to each of its points of local non-convexity. But this is not true for a closed connected 4- convex set which is neither convex nor starshaped. 
Theorem 3.10 Let $A$ be a closed connected 4 - convex subset in $E^{2}$, and let $L$ be a straight line in the plane. Then $L \cap A$ consists of at most three convex sets.

Proof. Let $A$ be a closed connected 4- convex subset in $E^{2}$, and let $L$ be a straight line in the plane. Suppose that $L \cap A$ has 4 - convex sets. Let $p_{1}, p_{2}, p_{3}$ and $p_{4}$ be four points each of them lie in a different piece of $L \bigcap A$, see Figure 4. It is obvious that no line segment $\left[p_{i} p_{j}\right]$ lies in $L \bigcap A$. But all of them lie in $L$ and so no one of them lie in $A$. This implies that $A$ is not a 4 - convex set which is a contradiction and the proof is complete.

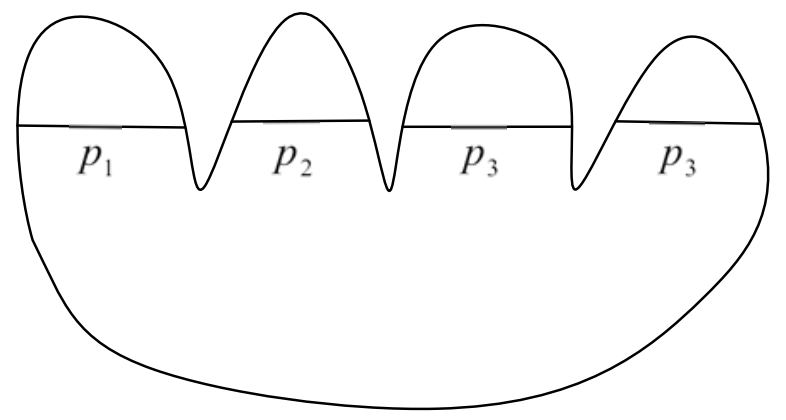

Figure 4: Intersection of a straight line and a set $A$

Let $A, B$ be two convex sets such that $B \subset A$ and $\partial A \cap \partial B=\phi$. Then $A \backslash B$ is not convex ( does not give any type of convexity ). Now, in the following theorem, we can remove a part of the interior of a convex set $A$ and the remaining part preserving a type of convexity.

Theorem 3.11 Let $A$ be a convex set in $E^{n}$ and let $p, q$ be two points in A. Then $A \backslash[p q]$ is a 3 - convex set.

Proof. Suppose that $A \backslash[p q]$ is not a 3- convex set. Then there are three points $a, b, c$ in $A \backslash[p q]$ such that no one of the line segments $[a b],[b c]$ and $[a c]$ is in $A \backslash[p q]$. This implies that the line segment $[p q]$ cuts the line segments $[a b],[b c]$ and $[a c]$. This shows that one of the points $a, b$ and $c$ must lie on $[p q]$ which is a contradiction and the proof is complete.

Theorem 3.12 Let $A$ be a convex set and let $p_{1}, p_{2}, p_{3}$ be three noncolinear points in $A$. Then $A \backslash T$ is a 4 - convex set, where $T$ is the convex hull of $p_{1}, p_{2}$ and $p_{3}$.

Proof. Let $a, b, c$ and $d$ be four points in $A \backslash T$. If one of these points (say a) does not lie in the 2 - plane $P$ determined by the points $p_{1}, p_{2}$ and $p_{3}$, then 
the line segment joining $a$ and any of the points $b, c$ or $d$ lies in $A$. Otherwise, all of the points $a, b, c$ and $d$ lie in $P$. In this case at least one of the straight lines $L\left(p_{1} \rightarrow p_{2}\right), L\left(p_{2} \rightarrow p_{3}\right)$ and $L\left(p_{1} \rightarrow p_{3}\right)$ separates two points (say $a$ and $b$ ) from $T$ in $P$. Hence $[a b]$ lies in $A \backslash T$ and so $A \backslash T$ is a 4 - convex set, and the proof is complete.

In the following, we use the concept of radial contraction and we try to get the relation between it and $m$ - convexity. The proof of the following lemma is in $[2]$.

Lemma 3.13 Let $A$ be a non-empty subset of $E^{n}$, and Let $x_{1}, x_{2} \in A$, with $C_{p}^{\lambda}\left(x_{1}\right)=\bar{x}_{1}$ and $C_{p}^{\lambda}\left(x_{2}\right)=\bar{x}_{2}$ for some $p \in A$ and some $\lambda \in(0,1)$. Then $C_{p}^{\lambda}\left(\left[x_{1} x_{2}\right]\right)=\left[\bar{x}_{1} \bar{x}_{2}\right]$.

Theorem 3.14 Let $A$ be a non-empty subset of $E^{n}$, and let $p \in E^{n}$ and $\lambda \in(0,1)$. Then $A$ is an $m$ - convex set if and only if $C_{p}^{\lambda}(A)$ is.

Proof. . First, suppose that $A$ is an $m$ - convex set. We want to prove that $C_{p}^{\lambda}(A)$ is an $m$ - convex set. Now, let $\bar{x}_{1}, \bar{x}_{2}, \ldots, \bar{x}_{m}$ be $m$ distinct points are in $C_{p}^{\lambda}(A)$. Then there are $m$ different points $x_{1}, x_{2}, \ldots, x_{m}$ in $A$ such that $C_{p}^{\lambda}\left(x_{i}\right)=$ $\bar{x}_{i}, 1 \leq i, j \leq m$. Since $A$ is an $m$ - convex set then at least one segment say $\left[x_{i} x_{j}\right]$ lies in $A, 1 \leq i, j \leq m$. By previous lemma, $C_{p}^{\lambda}\left(\left[x_{i} x_{j}\right]\right)=\left[\bar{x}_{i} \bar{x}_{j}\right] \subset C_{p}^{\lambda}(A)$ and $C_{p}^{\lambda}(A)$ must be an $m$ - convex set.

Conversely, suppose that $C_{p}^{\lambda}(A)$ is an $m$ - convex set. We want to prove that $A$ is an $m$-convex set. Let $x_{1}, x_{2}, \ldots, x_{m}$ be $m$ distinct points in $A$. Then there are $m$ points $\bar{x}_{1}, \bar{x}_{2}, \ldots, \bar{x}_{m}$ are in $C_{p}^{\lambda}(A)$, such that $C_{p}^{\lambda}\left(x_{i}\right)=\bar{x}_{i}, 1 \leq i, j \leq$ $m$. But $C_{p}^{\lambda}(A)$ is an $m$ - convex set, so there is a segment $\left[\bar{x}_{i} \bar{x}_{j}\right] \subset C_{p}^{\lambda}(A)$. Therefore, by previous lemma, $C_{p}^{\lambda}\left(\left[x_{i} x_{j}\right]\right)=\left[\bar{x}_{i} \bar{x}_{j}\right], 1 \leq i, j \leq m$. Hence, there exists a line segment $\left[x_{i} x_{j}\right]$ lies in $A$. So, $A$ must be an $m$ - convex set and the proof is complete.

Now we try to find a condition for a set to be $m$ - affine, and without loss of generality, we will consider the midpoint contraction.

Theorem 3.15 Let $p \in E^{n}$, and $A$ be an $m$-affine set. Then $C_{p}(A)$ is an $m$ - affine set.

Proof. Let $A$ be an $m$ - affine set. We want to prove that $C_{p}(A)$ is an $m$ affine set. So, let $\bar{x}_{1}, \bar{x}_{2}, \ldots, \bar{x}_{m}$ be $m$ distinct points in $C_{p}(A)$, then there are $x_{1}, x_{2}, \ldots, x_{m}$ in $A$ such that $C_{p}\left(x_{i}\right)=\bar{x}_{i}, 1 \leq i \leq m$. Since $A$ is $m$ - affine, then at least one of the straight lines (say $L\left(x_{i} \rightarrow x_{j}\right)$ ) passing through pairs of the points $x_{1}, x_{2}, \ldots, x_{m}$ lies in $A$. Then we claim that $C_{p}\left(L\left(x_{i} \rightarrow x_{j}\right)\right)=L\left(\bar{x}_{i} \rightarrow\right.$ $\left.\bar{x}_{j}\right)$ is a subset of $C_{p}(A)$ and hence $C_{p}(A)$ is $m$ - affine. Now we prove our claim. 
Let $\bar{z} \in L\left(\bar{x}_{i} \rightarrow \bar{x}_{j}\right)$, i.e., there exists $\alpha \in \Re$ such that $\bar{z}=\alpha \bar{x}_{i}+(1-\alpha) \bar{x}_{j}$. Now the point $z=\alpha x_{i}+(1-\alpha) x_{j} \in A$ and so

$$
\begin{aligned}
C_{p}(z) & =\frac{1}{2}(p+\alpha z) \\
& =\frac{1}{2}\left(p+\alpha x_{i}+(1-\alpha) x_{j}\right) \\
& =\alpha \bar{x}_{i}+(1-\alpha) \bar{x}_{j} \\
& =\bar{z} \in C_{p}(A) .
\end{aligned}
$$

Therefore, $C_{p}(A)$ is an $m$ - affine and the proof is complete.

\section{References}

[1] Beltagy, M. and El-Araby, A. On convex and starshaped hulls, kyungpook Mathematical Journal, 40(2000), 313-321.

[2] Beltagy, M. and Shenawy, S. A Note on convexity and starshapedness, Applied Mathematical Sciences, 4(2010), 2599-2608.

[3] Breen, M. Decomposition theorems for 3-convex subsets of the plane, Pacific Journal of Mathematics, 53(1974), 43-57.

[4] Breen, M. Decomposition for non-closed planar $m$-convex sets, Pacific Journal of Mathematics, 69(1977),317-324.

[5] Breen, M. A bound for decomposition of m-convex sets whose LNC points lie in a hyperplane, Proceeding of the American Mathematical Society, 72(1978), 159-162.

[6] Dwilewicz, R. J. A Short history of convexity, Differential geometryDynamical systems, 11(2009), 112-129.

[7] Kelly, P. J. and Weiss, M. L. Geometry and convexity, John Wiley and Sons, New York, 1978.

[8] Valentine, F. A. A Three point convexity property, Pacific Journal of Mathematics, 7(1957), 1227-1235.

\section{Received: March 17, 2013}

